\title{
Minimally Supported Frequency (MSF) $d$-Dilation Wavelets
}

\author{
Aparna Vyas ${ }^{1}$ and Gibak Kim ${ }^{2, *}$ (D) \\ 1 Department of Mathematics, Manav Rachna University, Faridabad 121004, India; aparna@mru.edu \\ 2 School of Electrical Engineering, Soongsil University, Seoul 06978, Korea \\ * Correspondence: imkgb27@ssu.ac.kr
}

check for updates

Citation: Vyas, A.; Kim, G. Minimally Supported Frequency (MSF) $d$-Dilation Wavelets. Mathematics 2021, 9, 1284.

https: / / doi.org/10.3390/math9111284

Academic Editor: Palle E.T. Jorgensen

Received: 12 March 2021

Accepted: 31 May 2021

Published: 3 June 2021

Publisher's Note: MDPI stays neutral with regard to jurisdictional claims in published maps and institutional affiliations.

\begin{abstract}
In this paper, we provide a geometric construction of a symmetric $2 n$-interval minimally supported frequency (MSF) $d$-dilation wavelet set with $d \in(1, \infty)$ and characterize all symmetric $d$-dilation wavelet sets. We also provide two special kinds of symmetric $d$-dilation wavelet sets, one of which has $4 m$-intervals whereas the other has $(4 m+2)$-intervals, for $m \in \mathbb{N}$. In addition, we construct a family of $d$-dilation wavelet sets that has an infinite number of components.
\end{abstract}

Keywords: $d$-dilation wavelet; MSF $d$-dilation wavelet; $d$-dilation wavelet set

\section{Introduction}

Wavelets are functions that are used to produce bases or frames by the dilates and translates of single function. Orthonormal wavelets and frames are widely used in applications such as signal and image processing, and sampling theory. A function $\psi$ in $L^{2}(\mathbb{R})$, whose successive dilates by $d>1$ and their integral translates form an orthonormal basis for $L^{2}(\mathbb{R})$, is called an orthonormal d-dilation wavelet for $L^{2}(\mathbb{R})$. A 2-dilation wavelet is simply called a wavelet. Chui and Shi [1] characterized the orthonormal wavelets and tight frames for dilation $d \in(1, \infty)$. An orthonormal d-dilation wavelet whose Fourier transform has the support to be of the smallest possible measure is called a minimally supported frequency (MSF) d-dilation wavelet. By an MSF wavelet, we mean a minimally supported frequency (MSF) 2-dilation wavelet. It is known that $\mid$ supp $\hat{\psi} \mid$, the Lebesgue measure of the Fourier transform of an orthonormal $d$-dilation wavelet $\psi$, is at least 1 . In fact, for an MSF $d$-dilation wavelet $\psi$, there is a measurable set $K$ of measure 1 such that $\hat{\psi}=\chi_{K}$. Set $K$ is called a d-dilation wavelet set. By a wavelet set, we mean a 2-dilation wavelet set [2,3]. Wavelet sets were first introduced by Dai and Larson [4]. Wavelet sets having certain number of components were studied by many researchers in [2,3,5-12]. Characterizations of two-, three-, and four-interval wavelet sets were obtained by Ha et al. for dilation 2 in [2]. A $d$-dilation wavelet set was characterized by Bownik et al. [7] as a measurable set of $\mathbb{R}$ that partitions $\mathbb{R}$ to be its integral translation and by its $d$-dilates. Further, Singh et al. [13] characterized a joint $(d,-d)$-dilation wavelet set and multiwavelet set in terms of wavelet-induced isomorphisms, where $|d|>1$.

Wavelets and wavelet sets were extensively studied, considering various aspects such as the multiresolution analysis and the path connectivity of wavelets, by several researchers in the field of wavelets [10-12,14,15]. From a result by Chui and Shi [1], according to which for a dilation $d$ such that $d^{j} \notin \mathbb{Q}$ for all $j \in \mathbb{N}$, the only wavelets that exist are MSF wavelets, the question of the existence or otherwise of a non-MSF wavelet for dilation other than the one considered by them arose. Bownik and Speegle [16] showed the existence of non-MSF wavelets for dilation $d>1$, for which there exists a $p \in \mathbb{Z} \backslash\{0\}$ such that $d^{p} \mathbb{Z} \cap \mathbb{Z} \neq\{0\}$. Therefore, constructing non-MSF wavelets became a matter of interest. Following a procedure originated from the work of Bownik and Speegle [16], we construct non-MSF wavelets for $L^{2}(\mathbb{R})[10,15,17]$ and $H^{2}(\mathbb{R})[17]$ for dilation 2, and their association with multiresolution analysis was considered. The path connectivity of certain subsets of the set of wavelets was also discussed [15]. 
Wavelets generated through wavelet sets are very important, as they are extensively used to produce examples in wavelet theory. Our main goal is to study the theory of MSF $d$-dilation wavelets and provide a geometric construction of MSF $d$-dilation wavelets for $L^{2}(\mathbb{R})$, where $d>1$, and characterize all symmetric $d$-dilation wavelet sets. Further, we provide finite and infinite families of $d$-dilation wavelets in $L^{2}(\mathbb{R})$.

Let $L^{1}(\mathbb{R})$ be the collection of all Lebesgue integrable functions on $\mathbb{R}$, and $L^{2}(\mathbb{R})$ be that of all Lebesgue square integrable functions on $\mathbb{R}$. Functions that are equal almost everywhere are identified. With the usual addition and scalar multiplication of functions together with inner product $\langle f, g\rangle$ of $f, g \in L^{2}(\mathbb{R})$ defined by

$$
\langle f, g\rangle=\int_{\mathbb{R}} f(x) \overline{g(x)} d x
$$

$L^{2}(\mathbb{R})$ becomes a Hilbert space. The Fourier transform is defined by

$$
\hat{f}(\xi)=\int_{\mathbb{R}} f(x) e^{-2 \pi i \xi x} d x
$$

where $f \in L^{1}(\mathbb{R}) \cap L^{2}(\mathbb{R})$. This uniquely extends to an operator on $L^{2}(\mathbb{R})$.

An orthonormal d-dilation wavelet is a function $\psi \in L^{2}(\mathbb{R})$ such that $\left\{\psi_{j, k}: j, k \in \mathbb{Z}\right\}$ is an orthonaormal basis for $L^{2}(\mathbb{R})$, where

$$
\psi_{j, k}(x)=d^{j / 2} \psi\left(d^{j} x-k\right)
$$

The $d$-dilation wavelet $\psi$ is called MSF d-dilation wavelet if there exists a set $K$ in $\mathbb{R}$, such that

$$
\hat{\psi}=\chi_{K}
$$

Set $K$ is called a $d$-dilation wavelet set, whose characterization is given by the following theorem:

Theorem 1. Let $K$ be a set in $\mathbb{R}$. $K$ is a d-dilation wavelet set iff the followings are satisfied:

(i) $\coprod_{n \in \mathbb{Z}}(K+n)=\mathbb{R}$ a.e.

(ii) $\coprod_{n \in \mathbb{Z}} d^{n} K=\mathbb{R}$ a.e.

Equivalently, $K$ is a $d$-dilation wavelet set if and only if $K$ is both translation-congruent to $[0,1]$ or $\left[-\frac{1}{2}, \frac{1}{2}\right]$ and $d$-dilation-congruent to $[-d b,-b] \cup[b, d b]$ for $b>0$.

As a consequence of Theorem 1, we have the following:

Corollary 1. Let $K$ and $W$ be subsets of $\mathbb{R}$, and $K$ be both translation- and d-dilation-equivalent to $W$. Then, $K$ is a d-dilation wavelet set if and only if $W$ is a d-dilation wavelet set.

The rest of the article is organized into two sections. Section 2 begins with a geometric construction of symmetric $2 n$-interval $d$-dilation wavelet set by following the technique provided by Arcozzi et al. [5] to obtain $2 n$-interval symmetric wavelet sets for dyadic dilation. The characterization of all symmetric $d$-dilation wavelet sets is also provided in Section 2. In addition, we provide a family of six-interval $d$-dilation wavelet sets and two special kinds of symmetric $d$-dilation wavelet sets, one of which has $4 m$ intervals, whereas the other has $(4 m+2)$ intervals, where $m \in \mathbb{N}$. In Section 3, we construct a family of $d$-dilation wavelet set that has an infinite number of components. The $d$-dilation wavelet set constructed in this section is bounded symmetric $d$-dilation wavelet sets having infinite number of components, and the accumulation point of these wavelet sets is their origin. MSF $d$-dilation wavelets arising from these $d$-dilation wavelet sets are also band-limited, and their Fourier transform is even and does not vanish in any neighborhood of origin, i.e., it was discontinuous at the origin. 


\section{Construction of Symmetric $d$-Dilation Wavelet Sets and $d$-dilation Wavelets}

In this section, we construct symmetric $2 n$-interval $d$-dilation wavelet sets with $d>1$ by following the method described in [5] to construct symmetric wavelet sets for dilation $d=2$ on the basis of the MSF polygonal. In addition, we provide a characterization of all symmetric $d$-dilation wavelet sets.

For $m_{j} \in \mathbb{N}_{0}=\mathbb{N} \bigcup\{0\}$ and $\lambda_{j} \in \mathbb{Z}$, consider set $D$ of all points $P_{j}=P\left[\lambda_{j}, m_{j}\right]=$ $\left(d^{-\lambda_{j}}, d^{-\lambda_{j} m_{j}}\right), \quad j=1,2, \ldots, n$ in the first quadrant of the Euclidean plane. For a finite set $\mathcal{P}=\left\{P_{1}, P_{2}, \ldots, P_{n}\right\}$ in $D$, the negative of the slope of the line joining $P_{j}$ and $P_{j+1}$ is

$$
-\frac{m_{j} d^{-\lambda_{j}}-m_{j+1} d^{-\lambda_{j+1}}}{d^{-\lambda_{j}}-d^{-\lambda_{j+1}}}
$$

denoted by $a_{j}$, where $j=1,2, \ldots, n-1$. Finite set $\mathcal{P}=\left\{P_{1}, P_{2}, \ldots, P_{n}\right\}$ is called $d$-MSF polygonal if

$$
\lambda_{1}=0,0=a_{0}<a_{1}<a_{2}<\ldots<a_{n}=\frac{1}{2},
$$

and

$$
d^{-\lambda_{n}}\left(2 m_{n}+1\right)=2 d m_{1} .
$$

Clearly, for $d=2$, the $d$-MSF polygonal is called the MSF polygonal.

Theorem 2. For a d-MSF polygonal $\mathcal{P}$ as defined above, let

$$
I_{j}=\left[a_{j-1}, a_{j}\right]+m_{j}, j=1,2, \ldots, n .
$$

Then, $K$ is a symmetric d-dilation wavelet set where $K=K^{-} \cup K^{+}$, with $K^{+}=\bigcup_{j=1}^{n} I_{j}$ and $K^{-}=-K^{+}$.

Proof. Since set $K$ is symmetric, for showing that $K$ is a $d$-dilation wavelet set, we only have to show that Conditions (i) and (ii) of Theorem 1 are satisfied by $K^{+}$for $\mathbb{R}^{+}$or $[0, \infty)$. It is obvious that $\amalg_{n \in \mathbb{Z}}\left(K^{+}+n\right)$ coincides a.e. with the set of real numbers that are congruent to a number in $[0,1 / 2]$, modulo $\mathbb{Z}$, if (6) holds. Next, let $H_{j}=d^{-\lambda_{j}} I_{j}, j=1,2, \ldots, n$. Using (5) and (7) we can show that $\amalg_{n \in \mathbb{Z}} d^{n} K^{+}=[0, \infty)$ a.e.. Hence, $K$ is a $d$-dilation wavelet set.

Remark 1. $K(\mathcal{P})$ is called d-dilation wavelet set associated to $\mathcal{P}$. If $\mathcal{P}_{1}$ and $\mathcal{P}_{2}$ are different polygonals i.e., $\mathcal{P}_{1} \neq \mathcal{P}_{2}$, then $K\left(\mathcal{P}_{1}\right) \neq K\left(\mathcal{P}_{2}\right)$.

The following example provides a family of six-interval symmetric d-dilation wavelet set for $L^{2}(\mathbb{R})$ with $d>1$.

Example 1. For $n=3$, let

$$
P_{1}=P\left(0, \frac{d^{s}}{2}(2 t+1)\right)=\left(1, \frac{d^{s}}{2}(2 t+1)\right), P_{2}=P(-v, 0)=\left(d^{v}, 0\right)
$$

and

$$
P_{3}=P(-s-1, t)=\left(d^{s+1}, t d^{s+1}\right),
$$

where $s, t$ and $v$ are non-negative integers such that $s \geq 1, t \geq 1$ and $v>s+1$. From these, we obtain

$$
a_{1}=\frac{d^{s}(2 t+1)}{2\left(d^{v}-1\right)}, \quad a_{2}=\frac{d^{s+1} t}{\left(d^{v}-d^{s+1}\right)} .
$$

Clearly, $a_{1}>0 . a_{1}<a_{2}$ and $a_{2}<a_{3}$ are satisfied if $1<2 t(d-1)$ and $d^{s+1}(2 t+1)<d^{v}$. Then $\mathcal{P}=\left\{P_{1}, P_{2}, P_{3}\right\}$ is a $d$-MSF polygonal for $d>1$. We have

$$
I_{1}=\left[\frac{d^{s}(2 t+1)}{2}, \frac{d^{s+v}(2 t+1)}{2\left(d^{v}-1\right)}\right], \quad I_{2}=\left[\frac{d^{s}(2 t+1)}{2\left(d^{v}-1\right)}, \frac{d^{s+1} t}{\left(d^{v}-d^{s+1}\right)}\right],
$$


and

$$
I_{3}=\left[\frac{d^{v} t}{\left(d^{v}-d^{s+1}\right)}, \frac{(2 t+1)}{2}\right] .
$$

Therefore, $K=K^{-} \cup K^{+}$with $K^{-}=-K^{+}$is a six-interval d-dilation wavelet set where

$$
K^{+}=\left[\frac{d^{s}(2 t+1)}{2}, \frac{d^{s+v}(2 t+1)}{2\left(d^{v}-1\right)}\right] \cup\left[\frac{d^{s}(2 t+1)}{2\left(d^{v}-1\right)}, \frac{d^{s+1} t}{\left(d^{v}-d^{s+1}\right)}\right] \cup\left[\frac{d^{v} t}{\left(d^{v}-d^{s+1}\right)}, \frac{(2 t+1)}{2}\right] .
$$

In this example, if we take $t=1, s=1, v=3$ and $d=4$, then we obtain a 4-dilation wavelet set $K=K^{-} \cup K^{+}$, where $K^{+}=\left[6, \frac{384}{63}\right] \cup\left[\frac{2}{21}, \frac{1}{3}\right] \cup\left[\frac{4}{3}, \frac{3}{2}\right]$ and $K^{-}=-K^{+}$.

Example 2. For $n=2$ and $d=2$, let $P_{1}=P\left(0,2^{r-1}\right)=\left(1,2^{r-1}\right), r \in \mathbb{N}$. Using (5), we can find $P_{2}=P(-r-1,0)=\left(2^{r+1}, 0\right)$. Then $\mathcal{P}=\left\{P_{1}, P_{2}\right\}$ is an MSF polygonal with $a_{1}=\frac{2^{r-1}}{2^{r+1}-1}$. Hence

$$
I_{1}=\left[2^{r-1}, \frac{2^{2 r}}{2^{r+1}-1}\right], I_{2}=\left[\frac{2^{r-1}}{2^{r+1}-1}, \frac{1}{2}\right] .
$$

Therefore, $K=K^{-} \cup K^{+}$is a four-interval wavelet set where $K^{+}=\left[2^{r-1}, \frac{2^{2 r}}{2^{r+1}-1}\right] \cup$ $\left[\frac{2^{r-1}}{2^{r+1}-1}, \frac{1}{2}\right]$ and $K^{-}=-K^{+}$. This family is the characterization of the four-interval wavelet set provided by Ha et al. in [2].

In the following theorem, we characterize all symmetric d-dilation wavelet sets with $d>1$, which are a finite union of intervals.

Theorem 3. Let $n \in \mathbb{N}$ and let $K \subset \mathbb{R}$ be a measurable set. $K$ is a symmetric d-dilation wavelet set with $d>1$ if and only if $K=K^{-} \cup K^{+}$with $K^{+}=\cup_{\alpha \in A} I_{\alpha}, A \subseteq\{1,2, \ldots, n\}$ and $K^{-}=-K^{+}$, where (6), and,

(a) $I_{j}=\left(\epsilon_{j}\left[a_{j-1}, a_{j}\right]+m_{j}\right)$, for $j=1,2, \ldots, n$, with $\epsilon_{j} \in\{-1,1\}, m_{j} \in \mathbb{N}_{0}$ and $a_{j}$ 's satisfying

(b) $\cup_{\alpha \in A} H_{\alpha}$ has the form $[b, d b]$ for some $b>0$ where $H_{\alpha}=d^{-\lambda_{\tau(\alpha)}} I_{\tau(\alpha)}, \alpha \in A, \lambda_{j} \in \mathbb{Z}, \tau$ is a permutation on $A$.

Proof. We only need to show that $K$ is translation-congruent to a subset of $[0,1]$ or $\left[-\frac{1}{2}, \frac{1}{2}\right]$ and $d$-dilation-congruent to $[-d b,-b] \cup[b, d b]$ for $b>0$. Clearly, the definition of $I_{j}^{\prime} s$ is equivalent to the condition that $K$ is translation-congruent to a subset of $[0,1]$ or $\left[-\frac{1}{2}, \frac{1}{2}\right]$, and the definition and conditions on $H_{\alpha}$ are equivalent to the condition for $K$ to be $d$ dilation-congruent to $[-d b,-b] \cup[b, d b]$ for $b>0$.

Now, we provide two special kinds of symmetric $d$-dilation wavelet sets with $d>1$, one of which has $4 m$-intervals, whereas the other has $(4 m+2)$-intervals, where $m \in \mathbb{N}$. The first is obtained by choosing an even positive integer, and the second by choosing an odd positive integer.

Example 3. Let $n \in 2 \mathbb{N}$ and $d \in(1, \infty)$. Define $\lambda_{j}$ 's and $m_{j}$ 's, where $j=1,2, \ldots, n$, as follows:

$$
\begin{aligned}
\lambda_{1}=0, \quad \lambda_{2 i} & =-(n-(i-2)), \text { for } i=1,2, \ldots, \frac{n}{2}, \\
\lambda_{2 i+1} & =-i, \text { for } i=1,2, \ldots, \frac{n}{2}-1,
\end{aligned}
$$

and

$$
\begin{gathered}
m_{1}=d^{n / 2}, m_{2 i}=0, \text { for } i=1,2, \ldots, \frac{n}{2} \\
m_{2 i+1}=d^{\frac{n}{2}-i}, \text { for } i=1,2, \ldots, \frac{n}{2}-1 .
\end{gathered}
$$


With the help of these $m_{j}$ 's and $\lambda_{j}{ }^{\prime}$ s, we obtain $P_{j}$, where $j=1,2, \ldots, n$, as follows:

$$
\begin{gathered}
P_{1} \equiv P\left[0, d^{\frac{n}{2}}\right]=\left(1, d^{\frac{n}{2}}\right), \\
P_{2 i} \equiv P[-(n-(i-2)), 0]=\left(d^{(n-(i-2))}, 0\right) \text { for } i=1,2, \ldots, \frac{n}{2},
\end{gathered}
$$

and

$$
P_{2 i+1} \equiv P\left[-i, d^{\frac{n}{2}-i}\right]=\left(d^{i}, d^{\frac{n}{2}}\right) \text { for } i=1,2, \ldots, \frac{n}{2}-1 .
$$

Thus, $a_{j}$, for $j=1,2, \ldots, n-1$, comes out to be

$$
a_{2 i-1}=\frac{d^{\frac{n}{2}}}{d^{n-i+2}-d^{i-1}} \text { for } i=1,2, \ldots, \frac{n}{2}
$$

and

$$
a_{2 i}=\frac{d^{\frac{n}{2}}}{d^{n-i+2}-d^{i}} \text { for } i=1,2, \ldots, \frac{n}{2}-1
$$

Therefore, positive side $\mathrm{K}^{+}$of the d-dilation wavelet set $K$ arises in the form of

$$
K^{+}=\left[d^{n / 2}, d^{n / 2}+\frac{d^{n / 2}}{d^{n+1}-1}\right] \cup\left[\frac{d^{n / 2}}{d^{n+1}-1}, \frac{d^{n / 2}}{d^{n+1}-2}\right] \cup \cdots \cup\left[\frac{d}{d^{3}-1}, \frac{1}{2}\right] .
$$

Hence, $K=K^{+} \cup K^{-}$where $K^{-}=-K^{+}$consists $4 m$ intervals, $m \in \mathbb{N}$.

Example 4. Let $n \in 2 \mathbb{N}+1$ and $d \in(1, \infty)$. Define $\lambda_{j}$ 's and $m_{j}{ }^{\prime} s$, where $j=1,2, \ldots, n$, as follows:

$$
\begin{gathered}
\lambda_{1}=0, \quad \lambda_{2 i}=-4-\left(\frac{n-2 i+1}{2}\right) \text { for } i=1,2, \ldots, \frac{n-1}{2}, \\
\lambda_{2 i+1}=\frac{n-2 i-7}{2} \text { for } i=1,2, \ldots, \frac{n-1}{2},
\end{gathered}
$$

and

$$
\begin{gathered}
m_{1}=6, m_{2 i}=0 \text { for } i=1,2, \ldots, \frac{n-1}{2}, \\
m_{2 i+1}=d^{\frac{n-2 i-1}{2}} \text { for } i=1,2, \ldots, \frac{n-1}{2} .
\end{gathered}
$$

With the help of these $m_{j}$ 's and $\lambda_{j}$ 's, we obtain $P_{j}$, where $j=1,2, \ldots, n$, as follows:

$$
\begin{gathered}
P_{1} \equiv P[0,6]=(1,6), \\
P_{2 i} \equiv P\left[-4-\left(\frac{n-2 i+1}{2}\right), 0\right]=\left(d^{4+\left(\frac{n-2 i+1}{2}\right)}, 0\right) \text { for } i=1,2, \ldots, \frac{n-1}{2},
\end{gathered}
$$

and

$$
P_{2 i+1} \equiv P\left[\frac{n-2 i-7}{2}, d^{\frac{n-2 i-1}{2}}\right]=\left(d^{-\frac{n-2 i-7}{2}}, d^{3}\right) \text { for } i=1,2, \ldots, \frac{n-1}{2} .
$$

Thus $a_{j}$, for $j=1,2, \ldots, n-1$, comes out to be

$$
a_{1}=\frac{6}{d^{\frac{n+7}{2}}-1}, \quad a_{2 i}=\frac{d^{\frac{n-1}{2}-i}}{d^{n-2 i+1}-1} \text { for } i=1,2, \ldots, \frac{n-1}{2},
$$

and

$$
a_{2 i+1}=\frac{d^{\frac{n-1}{2}-i}}{d^{n-2 i}-1} \text { for } i=1,2, \ldots, \frac{n-3}{2}
$$


Therefore, positive side $K^{+}$of the d-dilation wavelet set $K$, arises in the form of

$$
K^{+}=\left[6,6+\frac{6}{d^{\frac{n+7}{2}}-1}\right] \cup\left[\frac{6}{d^{\frac{n+7}{2}}-1}, \frac{d^{\frac{n-3}{2}}}{d^{n-1}-1}\right] \cup \cdots \cup\left[\frac{d^{2}}{d^{2}-1}, \frac{3}{2}\right] .
$$

Hence, $K=K^{+} \cup K^{-}$where $K^{-}=-K^{+}$consists $(4 m+2)$ intervals, $m \in \mathbb{N}$.

The symmetric $d$-dilation wavelet sets constructed in this section have a finite number of components. By employing the method of Brandolini et al. [18], we construct two families of $d$-dilation wavelet sets by using Examples 3 and 4 in the following section, which has an infinite number of components. MSF $d$-dilation wavelets arising from these $d$-dilation wavelet sets are also band-limited, of which the Fourier transforms are even and does not vanish in any neighborhood of the origin.

\section{Symmetric $d$-Dilation Wavelet Sets Having Infinite Number of Components}

With the help of the $d$-dilation wavelet sets obtained Examples 3 and 4, we provide families of bounded symmetric $d$-dilation wavelet sets having infinite number of components and the origin as their accumulation point.

Consider $d$-dilation wavelet set $K=K^{-} \cup K^{+}$with $K^{-}=-K^{+}$, constructed in Example 3 and $\mathrm{K}^{+}=I_{1}^{+} \cup I_{2}^{+} \cup \cdots \cup I_{n}^{+}$where

$$
I_{1}^{+}=\left[d^{n / 2}, d^{n / 2}+\frac{d^{n / 2}}{d^{n+1}-1}\right] I_{2}^{+}=\left[\frac{d^{n / 2}}{d^{n+1}-1}, \frac{d^{n / 2}}{d^{n+1}-2}\right]
$$

and

$$
I_{n}^{+}=\left[\frac{d}{d^{3}-1}, \frac{1}{2}\right]
$$

Selecting $b_{n}=\frac{d^{n / 2}}{d^{n+1}-1}$, we consider the following intervals:

$$
S_{1}=\left[\frac{b_{n}}{d}+\frac{\epsilon}{d^{n+1}}, \frac{b_{n}}{d}+\epsilon\right], \quad S_{2}=\left[b_{n}+d \epsilon, \frac{d^{n / 2}}{d^{n+1}-2}\right],
$$

and

$$
S_{3}=\left[d^{n+1} b_{n}, d^{n+1} b_{n}+d \epsilon\right] .
$$

Since $\epsilon \in\left(0, \delta_{n}\right), S_{2}$ is a nonempty set. Setting $E_{0}=S_{1}+d^{n / 2}, F_{0}=\frac{1}{d^{n+2}} E_{0}$, and for $r \geq 1, \quad E_{r}=F_{r-1}+d^{n / 2}, \quad F_{r}=\frac{1}{d^{r+n+2}} E_{r}$, we denote

$$
\left(I_{1}^{+}-\bigcup_{r=0}^{\infty} E_{r}\right) \cup\left(\bigcup_{r=0}^{\infty} F_{r}\right) \cup\left(S_{1} \cup S_{2} \cup S_{3}\right) \cup I_{3}^{+} \cup I_{4}^{+} \cup \cdots \cup I_{n}^{+}
$$

by $K_{\epsilon}^{+}$, and define

$$
K_{\epsilon}=K_{\epsilon}^{-} \cup K_{\epsilon}^{+}, \text {where } K_{\epsilon}^{-}=-K_{\epsilon}^{+} .
$$

To prove that $K_{\epsilon}$ is a $d$-dilation wavelet set, we make use of Corollary 1, according to which $K_{\epsilon}$ is to be shown to be translation- and $d$-dilation-equivalent to a $d$-dilation wavelet set, in general, and hence to the $d$-dilation wavelet set $K$ constructed in Example 3, in particular. On account of the symmetry of $d$-dilation wavelet sets, it suffices to show that $K_{\epsilon}^{+}$is both translation- and $d$-dilation-equivalent to $K^{+}$.

First, by induction, we obtain that $E_{r} \subset I_{1}^{+}$, for all $r \geq 0$. Observing that

$$
b_{n}+d^{n / 2}=d^{n+1} b_{n}
$$

we have

$$
\left[0, b_{n}\right]+d^{n / 2}=\left[d^{n / 2}, d^{n+1} b_{n}\right]=I_{1}^{+}
$$


and hence

$$
E_{0}=S_{1}+d^{n / 2} \subset\left[0, b_{n}\right]+d^{n / 2}=I_{1}^{+} .
$$

Now, assume that $E_{m} \subset I_{1}^{+}$. Then,

$$
F_{m}=d^{-(m+n+2)} E_{m} \subset d^{-(m+1)}\left[0, b_{n}\right] \subset\left[0, b_{n}\right],
$$

and hence

$$
E_{m+1}=F_{m}+d^{n / 2} \subset\left[0, b_{n}\right]+d^{n / 2}=I_{1}^{+} .
$$

As intervals $E_{r}, r \geq 0$ lie inside the interval $I_{1}^{+}$, and $E_{r+1}$ lies to the left of $E_{r}$, for all $r \geq 0, F_{r+1}$ lies to the left of $F_{r}$, for all $r \geq 0$.

Because sets $I_{3}^{+}, I_{4}^{+}, \ldots, I_{n}^{+}$appear in both the partitions of $K_{\epsilon}^{+}$and of $K^{+}$, that $K_{\epsilon}^{+}$is $d$-dilation- and translation-equivalent to $K^{+}$follows from (A) and (B), respectively.

(A) (i) $\frac{1}{d^{n+1}} S_{3} \cup d S_{1} \cup S_{2}$

$$
\begin{aligned}
& =\left[b_{n}, b_{n}+\frac{d \epsilon}{d^{n+1}}\right] \cup\left[b_{n}+\frac{d \epsilon}{d^{n+1}}, b_{n}+d \epsilon\right] \cup\left[b_{n}+d \epsilon, \frac{d^{n / 2}}{d^{n+1}-2}\right] \\
& =\left[b_{n}, \frac{d^{n / 2}}{d^{n+1}-2}\right]=I_{2}^{+},
\end{aligned}
$$

(ii) $\quad\left(I_{1}^{+}-\bigcup_{r=0}^{\infty} E_{r}\right) \cup\left(\bigcup_{r=0}^{\infty} d^{r+n+2} F_{r}\right)$

(B) (i) $\left(S_{3}-d^{n / 2}\right) \cup S_{2}$

$$
=\left(I_{1}^{+}-\bigcup_{r=0}^{\infty} E_{r}\right) \cup\left(\bigcup_{r=0}^{\infty} E_{r}\right)=I_{1}^{+} \text {. }
$$

$$
\begin{aligned}
& =\left[b_{n}, b_{n}+d \epsilon\right] \cup\left[b_{n}+d \epsilon, \frac{d^{n / 2}}{d^{n+1}-2}\right] \\
& =\left[b_{n}, \frac{d^{n / 2}}{d^{n+1}-2}\right]=I_{2}^{+},
\end{aligned}
$$

(ii) $\quad\left(I_{1}^{+}-\bigcup_{r=0}^{\infty} E_{r}\right) \cup\left(\cup_{r=0}^{\infty}\left(F_{r}+d^{n / 2}\right)\right) \cup\left(S_{1}+d^{n / 2}\right)=I_{1}^{+}$.

Further, since a neighborhood of the origin intersects $\cup_{r=0}^{\infty} F_{r}$, the origin is an accumulation point of the $d$-dilation wavelet set $K_{\epsilon}$. Now, we have the following result:

Theorem 4. For $n \in 2 \mathbb{N}, d \in(1, \infty)$ and $\epsilon \in\left(0, \delta_{n}\right)$, where $\delta_{n}=\frac{d^{n / 2}}{d\left(d^{n+1}-2\right)\left(d^{n+1}-1\right)}$, there exists a bounded symmetric d-dilation wavelet set $K_{\epsilon}$ having infinite number of components and the origin as an accumulation point.

Again, consider the $d$-dilation wavelet set $K=K^{-} \cup K^{+}$with $K^{-}=-K^{+}$constructed in Example 4 and $\mathrm{K}^{+}=I_{1}^{+} \cup I_{2}^{+} \cup \cdots \cup I_{n}^{+}$where

$$
I_{1}^{+}=\left[6,6+\frac{6}{d^{\frac{n+7}{2}}-1}\right] I_{2}^{+}=\left[\frac{6}{d^{\frac{n+7}{2}}-1}, \frac{d^{\frac{n-3}{2}}}{d^{n-1}-1}\right] \text { and } I_{n}^{+}=\left[\frac{d^{2}}{d^{2}-1}, \frac{3}{2}\right] .
$$

With $b_{n}=\frac{6}{d^{\frac{n+7}{2}}-1}$, we consider the following intervals:

$$
S_{1}=\left[\frac{b_{n}}{d}+\frac{\epsilon}{d^{\frac{n+7}{2}}}, \frac{b_{n}}{d}+\epsilon\right], \quad S_{2}=\left[b_{n}+d \epsilon, \frac{d^{\frac{n-3}{2}}}{d^{n-1}-1}\right],
$$

and

$$
S_{3}=\left[d^{\frac{n+7}{2}} b_{n}, d^{\frac{n+7}{2}} b_{n}+d \epsilon\right],
$$

where $S_{2}$ is a nonempty set that follows on account of the choice of $\epsilon$. Setting $E_{0}=$ $S_{1}+6, \quad F_{0}=\frac{1}{d^{\frac{n+9}{2}}} E_{0}$, and for $r \geq 1, \quad E_{r}=F_{r-1}+6, \quad F_{r}=\frac{1}{d^{r+\frac{n+9}{2}}} E_{r}$, we denote

$$
\left(I_{1}^{+}-\cup_{r=0}^{\infty} E_{r}\right) \cup\left(\cup_{r=0}^{\infty} F_{r}\right) \cup\left(S_{1} \cup S_{2} \cup S_{3}\right) \cup I_{3}^{+} \cup I_{4}^{+} \cup \cdots \cup I_{n}^{+},
$$

by $K_{\epsilon}^{+}$. Then

$$
K_{\epsilon}=K_{\epsilon}^{-} \cup K_{\epsilon}^{+}, \text {where } K_{\epsilon}^{-}=-K_{\epsilon}^{+},
$$


is the required $d$-dilation wavelet set. To obtain that $K_{\epsilon}$ is a $d$-dilation wavelet set, we show that $K_{\epsilon}^{+}$is both translation- and $d$-dilation-equivalent to $K^{+}$constructed in Example 4 . Using induction, we obtain $E_{r} \subset I_{1}^{+}$, for all $r \geq 0$. In fact, from

$$
b_{n}+6=d^{\frac{n+7}{2}} b_{n}
$$

it follows that

$$
\left[0, b_{n}\right]+6=\left[6, d^{\frac{n+7}{2}} b_{n}\right]=I_{1}^{+},
$$

and hence

$$
E_{0}=S_{1}+6 \subset\left[0, b_{n}\right]+6=I_{1}^{+} .
$$

Next, assuming that $E_{m} \subset I_{1}^{+}$, we have

$$
F_{m}=d^{-\left(m+\frac{n+9}{2}\right)} E_{m} \subset d^{-(m+1)}\left[0, b_{n}\right] \subset\left[0, b_{n}\right],
$$

and hence

$$
E_{m+1}=F_{m}+6 \subset\left[0, b_{n}\right]+6=I_{1}^{+} .
$$

As intervals $E_{r}, r \geq 0$ lie inside the interval $I_{1}^{+}$, and $E_{r+1}$ lies to the left of $E_{r}$, for all $r \geq 0, F_{r+1}$ lies to the left of $F_{r}$, for all $r \geq 0$.

Because sets $I_{3}^{+}, I_{4}^{+}, \ldots, I_{n}^{+}$appear in both partitions of $K_{\epsilon}^{+}$and $K^{+}$, that $K_{\epsilon}^{+}$is $d$-dilationand translation-equivalent to $K^{+}$follows from (A) and (B), respectively.

(A) (i) $\frac{1}{d^{\frac{n+7}{2}}} S_{3} \cup d S_{1} \cup S_{2}$

$$
\begin{aligned}
& =\left[b_{n}, b_{n}+\frac{d \epsilon}{d^{\frac{n+7}{2}}}\right] \cup\left[b_{n}+\frac{d \epsilon}{d^{\frac{n+7}{2}}}, b_{n}+d \epsilon\right] \cup\left[b_{n}+d \epsilon, \frac{d^{\frac{n-3}{2}}}{2^{n-1}-1}\right] \\
& =\left[b_{n}, \frac{d^{\frac{n-3}{2}}}{d^{n-1}-1}\right]=I_{2}^{+}
\end{aligned}
$$

(ii) $\left(I_{1}^{+}-\bigcup_{r=0}^{\infty} E_{r}\right) \cup\left(\bigcup_{r=0}^{\infty} d^{r+\frac{n+9}{2}} F_{r}\right)$

$$
=\left(I_{1}^{+}-\bigcup_{r=0}^{\infty} E_{r}\right) \cup\left(\bigcup_{r=0}^{\infty} E_{r}\right)=I_{1}^{+} .
$$

(B) $\quad$ (i) $\left(S_{3}-6\right) \cup S_{2}=\left[b_{n}, b_{n}+d \epsilon\right] \cup\left[b_{n}+d \epsilon, \frac{d^{\frac{n-3}{2}}}{d^{n-1}-1}\right]=\left[b_{n}, \frac{d^{\frac{n-3}{2}}}{d^{n-1}-1}\right]=I_{2}^{+}$,

(ii) $\left(I_{1}^{+}-\bigcup_{r=0}^{\infty} E_{r}\right) \cup\left(\bigcup_{r=0}^{\infty}\left(F_{r}+6\right)\right) \cup\left(S_{1}+6\right)=I_{1}^{+}$.

Therefore, $K_{\epsilon}$ is a $d$-dilation wavelet set. Further, since a neighborhood of the origin intersects $\cup_{r=0}^{\infty} F_{r}$, the origin is an accumulation point of the $d$-dilation wavelet set $W_{\epsilon}$. Now, we have the following result:

Theorem 5. For $n \in 2 \mathbb{N}+1, d \in(1, \infty)$ and $\epsilon \in\left(0, \delta_{n}\right)$, there exists a bounded symmetric $d$-dilation wavelet set $K_{\epsilon}$ having infinite number of components and the origin as an accumulation point, where $\delta_{n}=\frac{d^{\frac{n-5}{2}}\left(d^{\frac{n+1}{2}}\left(d^{3}-6\right)-1\right)+6}{\left(d^{n-1}-1\right)\left(d^{\frac{n+7}{2}}-1\right)}$.

Remark 2. Let $\hat{\psi}_{\epsilon}$ be the characteristic function on set $K_{\epsilon}$, constructed in Theorems 4 and 5 , respectively. Then, $\psi_{\epsilon}$ is a band-limited d-dilation wavelet such that $\hat{\psi}_{\epsilon}$ is even and does not vanish in any neighborhood of the origin. In particular, it is discontinuous at the origin.

Remark 3. Employing Examples 2-4, we can construct non-MSF d-dilation wavelets with $d>1$ for $L^{2}(\mathbb{R})$. The technique of constructing such wavelets is similar to the one utilized in $[10,15,17]$. Most of the constructed non-MSF d-dilation wavelets were non-MRA. 


\section{Summary}

In this paper, we studied the theory of MSF $d$-dilation wavelets and provided a geometric construction of MSF $d$-dilation wavelet set with characterization of all symmetric $d$-dilation $(d \in(1, \infty))$ wavelet sets. For the geometric construction of wavelet sets, we exploited Arcozzi's technique [5] to obtain $2 n$-interval symmetric wavelet sets for dyadic dilation. We also provided a family of six-interval $d$-dilation wavelet sets and two special kinds of symmetric $d$-dilation wavelet set. The two symmetric $d$-dilation wavelets had $4 m$-intervals and $(4 m+2)$-intervals $(m \in \mathbb{N})$, respectively. Further, we constructed infinite families of $d$-dilation wavelets that were bounded symmetric $d$-dilation wavelet sets. The MSF $d$-dilation wavelets on these wavelet sets were band-limited, and their characteristic function was even and discontinuous at the origin.

Author Contributions: Conceptualization, A.V.; Formal analysis, A.V.; Supervision, G.K. All authors have read and agreed to the published version of the manuscript.

Funding: This work was supported by an Information and Communication Technology Promotion grant from the Korean Government (B0101-1-0525).

Institutional Review Board Statement: Not applicable

Informed Consent Statement: Not applicable

Data Availability Statement: Not applicable

Conflicts of Interest: The authors declare no conflict of interest

\section{References}

1. Chui, C.K.; Shi, X. Orthonormal wavelets and tight frames with arbitrary real dilations. Appl. Comput. Harmon. Anal. 2000, 9, 243-264. [CrossRef]

2. Ha, Y.H.; Kang, H.; Lee, J.; Seo, J.K. Unimodular wavelets for $L^{2}$ and the Hardy space $H^{2}$. Mich. Math. J. 1994, 41, 345-361. [CrossRef]

3. Hernandez, E.; Weiss, G. A First Course on Wavelets; CRC Press: Boca Raton, FL, USA, 1996.

4. Dai, X.; Larson, D. Wandering Vectors for Unitary Systems and Orthogonal Wavelets; American Mathematical Society: Providence, RI, USA, 1998; Volume 134.

5. Arcozzi, N.; Behera, B.; Madan, S. Large classes of minimally supported frequency wavelets of $L^{2}(\mathbb{R})$ and $H^{2}(\mathbb{R})$. J. Geom. Anal. 2003, 13, 557-579. [CrossRef]

6. Bownik, M.; Hoover, K.R. Dimension functions of rationally dilated GMRA's and wavelets. J. Fourier Anal. Appl. 2009, 15, 579-585. [CrossRef]

7. Bownik, M.; Rzeszotnik, Z.; Speegle, D. A characterzation of dimension functions of wavelets. Appl. Comput. Harmon. Anal. 2001, 10, 71-92. [CrossRef]

8. Dai, X.; Larson, D.; Speegle, D. Wavelet sets in $\mathbb{R}^{n}$. J. Fourier Anal. Appl. 1997, 3, 451-456. [CrossRef]

9. Merill, K.D. Simple wavelet sets for scalar dilations. In $\mathbb{R}^{2}$, Representations, Wavelets and Frames, a Celebration of the Mathematical Work of L. W. Baggett; Birkhauser: Boston, MA, USA, 2009; pp. 177-192.

10. Vyas, A. On Multiresolution Analysis. Ph.D. Thesis, University of Allahabad, Allahabad, India, 2010.

11. Vyas, A.; Kim, G. Non-MSF MRA Wavelets. Int. J. Pure Appl. Math. 2017, 115, 1-12. [CrossRef]

12. Bownik, M.; Rzeszotnik, Z. Open Problems in Wavelet Theory. In Operator Theory, Operator Algebras and Their Interactions with Geometry and Topology; Birkhauser: Boston, MA, USA, 2020; pp. 77-100.

13. Singh, P.; Masood, D. On wavelet induced isomorphisms for joint $(d,-d)$-dilation wavelet and multiwavelet sets. Int. J. Wavelets Multiresolut. Inf. Process. 2015, 13. [CrossRef]

14. Speegle, D.M. The s-elementary wavelets are path connected. Proc. Amer. Math. Soc. 1999, 127, 223-233. [CrossRef]

15. Vyas, A.; Dubey, R. Non-MSF wavelets from six interval MSF wavelets. Int. J. Wavelets Multiresolut. Inf. Process. 2011, 9, 375-385. [CrossRef]

16. Bownik, M.; Speegle, D. The Wavelet Dimension Function for Real Dilations and Dilations Admitting Non-MSF Wavelets, Approximation Theory X: Wavelets, Splines and Applications; Vanderbilt University Press: Nashville, TN, USA, 2002; pp. 63-85.

17. Vyas, A. Construction of non-MSF non-MRA wavelets for $L^{2}(\mathbb{R})$ and $H^{2}(\mathbb{R})$ from MSF wavelets. Bull. Pol. Acad. Sci. Math. 2009, 57, 33-40. [CrossRef]

18. Brandolini, L.; Garrigo's, G.; Rzeszotnik, Z.; Weiss, G. The behaviour at the origin of a class of band-limited wavelets. Contemp. Math. 1999, 247, 75-91. 